\title{
Update in pediatric renal sonography: focus on hyperechoic kidneys
}

\author{
Fred Avni
}

(C) Springer-Verlag 2011

For years, US has been the primary modality for monitoring growth and development of the kidneys before and after birth. Good knowledge of the normal imaging appearances of the kidney is mandatory in order to diagnose renal disease. One of the main objectives of sonography is to detect and evaluate congenital uropathies. The definition of urinary tract dilatation is standardized, and the role of US is essential and clearly defined for this diagnosis. Still, we need long-term follow-up in order to correlate the degree of dilatation and renal function. More recently, thanks to highdefinition transducers, more attention has been given to characterization of nephropathies through more careful analysis of the renal cortex and medulla.

Cortico-medullary differentiation (CMD; determined by the different tissue characteristics of the cortex and medulla) is a well-established characteristic of renal sonography in neonates and young children. The CMD can be visualized already around 18-20 weeks' gestation. In the fetus, the thickness of both cortex and medulla increases progressively throughout pregnancy; yet medullary thickness increases more rapidly than cortical thickness, and therefore the cortico-medullary ratio decreases (from 0.5 around 20 weeks' to 0.4 around 32 weeks' gestation). Notably, the overall echogenicity of the fetal

$\overline{\text { Disclaimer Dr. Fred Avni has no financial interests, investigational or }}$ off-label uses to disclose.

F. Avni $(\bowtie)$

Department of Radiology, Hôpital Universitaire Des Enfants

Reine Fabiola,

1020, Brussels, Belgium

e-mail: favni@ulb.ac.be renal parenchyma decreases with time. It is hyperechoic relative to the liver or spleen during the second trimester and becomes relatively iso- or hypo-echoic after 32 weeks' gestation. Based on these normal references, it is possible to diagnose various nephropathies prenatally (polycystic kidney diseases, nephrotic syndrome, renal vein thrombosis, etc).

After birth, there is a massive fluid overload in the kidneys, and this renders the renal parenchyma relatively hyperechoic. This feature is more marked in prematures, but can be observed in term neonates. As mentioned above, CMD is always visualized. The cortico-medullary ratio should be around 0.38. An abnormal ratio can be observed in various conditions. For instance, in smallfor-dates newborns it is decreased to 0.30 , whereas in diseases such as congenital nephrotic syndrome, the pyramids will appear small and irregular and the CMD will be difficult to assess.

The sonographic pattern of the CMD has become an essential feature for analysis. The CMD can be increased, absent or reversed, and each pattern will suggest a list of differential diagnoses. Reversed CMD (with hyperechoic medulla) or areas of increased echogenicity in the kidney is referred to as "nephrocalcinosis", and has been the focus of much research. Medullary nephrocalcinosis is the commonest form; it has been linked to hypercalciuria and metabolic diseases. For some, genetic studies are mandatory in order to distinguish between the diagnostic possibilities. Hyperoxaluria type I, proximal tubular acidosis and Dent syndrome are among other diseases that can be diagnosed by the sonographic patterns.

Cortical nephrocalcinosis is less frequent. Distinctive patterns of localized or diffuse areas of calcification may 
lead to several specific diagnoses once clinical and biological data are integrated. Diffuse cortical hyperechogenicity may result from some metabolic diseases associated with hypercalciuria. Localized areas of calcification may result from vascular insults. These have to be differentiated from renal dysplasia or renal cystic disease.
We have surely progressed in the characterization of nephropathies thanks to sonography. Yet, as we expand the use of focused US, we depict more and more, often unexplainable, altered echogenicity, especially in and around the pyramids. Therefore, more work is required in order to define the role of sonography, and to establish the validity of more subtle sonographic findings. 